\section{Choosing the Best Camera for Fluorescence Microscopy}

Dr.Alan Mullan: Product Specialist for Microscopy Cameras at Andor Technology, Dr.Aleksandra Marsh:Technical Author and Copywriter at Andor Technology

Choosing the most suitable camera for fluorescence $\mid$ cameras featuring back-illuminated sensors has microscopy can appear to be a daunting task when become available, promising greater sensitivity than faced with the variety of camera technologies and models on offer. The technical specifications front-illuminated sensors. The increase in sensitivity provided may also be difficult to understand coupled with large sensor sizes and speed that and relate to how they will affect imaging for any $\mathrm{sCMOS}$ cameras are already known for make an given application. In recent times the scientific attractive case to many fluorescence microscopy Complementary Metal-Oxide Semiconductor applications. But are they always more suitable? cameras (sCMOS) and Electron Multiplying Charge And how do they compare to EMCCD which has Coupled Device (EMCCD) have been the two main continued to remain the detector of choice for the camera technologies available to the life science most demanding low light applications? researcher. Meanwhile the older interline Charge Coupled Device (CCD) cameras that were once widely used have now largely been displaced. sCMOS cameras are known for their large fields of view an high frame rate performance, while EMCCD-based cameras offer the greatest sensitivity, down to the single photon level. However, it should be noted that EMCCD cameras are often seen as a premium option due to their higher cost.

Since our earlier article in the December 2014 edition of infocus, a new generation of sCMOS

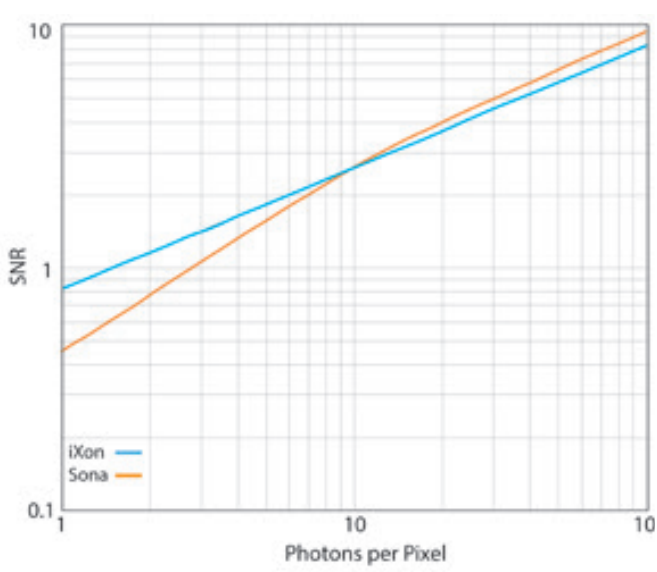

Figure I:A comparison of the signal to noise ratio (SNR) against (Sona) and back-illuminated EMCCD (iXon).

\section{Key parameters to consider:}

For fluorescence microscopy there are three key parameters that need to be considered:

Sensitivity: How sensitive is the camera? i.e. a measure of the ability to detect photons emitted from fluorophores

Field of View:The size of the sample area that can be seen (and resolved) in a single field of view

Speed: How well can the camera resolve temporal information from dynamic events?

The importance of each of these parameters will vary depending on the specific application, howeve sensitivity is often the primary consideration. We will look at cameras summarised in Table I as examples of the different camera types available, but first, let's look at the different camera technologies.

\section{Competing Camera \\ Technologies}

\section{EMCCD}

Cameras based on EMCCD sensors were introduced in $200 \mathrm{I}$ and represented a significant leap forward compared to standard CCD cameras, combining ultra-sensitivity with speed. Central to an EMCCD
sCMOS

sCMOS cameras were first introduced in 2010 by Andor and other camera companies as a compelling alternative solution to the aging interline CCD sensor-based cameras. SCMOS-based cameras rapidly became the technology of choice for many applications across both life and physical sciences. The popularity of sCMOS-based cameras over other camera technologies (CCD and EMCCD) is largely due to their very useful set of attributes for microscopy imaging: low noise, large sensor sizes while achieving fast frame rates. There are now many models available, typically at lower price points than EMCCD cameras, meaning that there is an SCMOS camera option for most research budgets.

There have been several refinements to the sensitivity of SCMOS sensors that have seen quantum efficiency (QE) jump from 60 to $82 \%$,

Figure 2: Comparative low light images taken with Andor Zyla frontilluminated sCMOS ( 1.4 electrons read noise @ $560 \mathrm{MHz}$ ) vs Andor iXon 888 back-illuminated EMCCD ( < le- read noise), under a range of photon intensity conditions plots. SCMOS was $2 \times 2$ binned in order to have the same effective pixel pitch (and light collection area per of back-illuminated EMCCD under extremely low light conditions is readily apparent.

is an on-chip amplification that multiplies even single photon events well above the read noise floor by a mechanism termed "impact ionization". Thus, EMCCDs are capable of single photon sensitivity at high frame rates. This established EMCCD as the camera of choice in applications demanding ultralow light measurements, such as single molecule detection and photon counting experiments. While a well optimized EMCCD can be thought of as having a "zero noise floor", there is a downside to this technology. The EM amplification carries an additional noise source called multiplicative noise (sometimes called EM noise or Excess Noise Factor). EM noise effectively increases the shot noise (or Poisson noise) of the signal by a factor of $x \mid$. 41 . This is observed as an increase in both pixel-to-pixel and frame-to-frame signal variability. EMCCD cameras also tend to have larger pixel sizes (e.g. 13 or 16 $\mu \mathrm{m}$ ) which are good for sensitivity at low light levels, but less suited to resolution at lower objective magnifications. edging their sensitivity closer towards that of EMCCD. Until recently, sCMOS sensors have been of the front-illuminated type whereby photons must pass through the bulk silicon and the electrode structure to reach the photosensitive area of the sensor and create charge. Therefore, a portion of the light is absorbed or reflected and so reduces the efficiency of collection and conversion of photons into electrons. Back-illuminated sensors provide higher $\mathrm{QE}$ as obstructions to incoming light have been placed behind the photosensitive area. Backilluminated CCD and EMCCD sensors have been available for some time, but it is only recently that SCMOS sensors suitable for microscopy have benefited from back-illuminated sensors. One advantage of this is a higher QE, for example, the ew Andor Sona 4.2B-II sCMOS camera has a QE of $95 \%$ which is comparable to that of EMCCD at the emission wavelengths of common fluorophores such as GFP.

\section{Sensitivity} importance when considering the sensitivity of any imaging sensor. This defines the ability of the camera to detect a signal and distinguish it from
The Signal-to-Noise Ratio (SNR) is of key 
the surrounding noise. The Signal to Noise ratio for a given light level at a specific wavelength can be described by the equation:

$$
S N R=\frac{S}{\sqrt{S+\left[N D \times T^{2}\right]+N R^{2}}}
$$

Where:

$\mathrm{S}=$ The signal level arriving at the detector (electrons) $\mathrm{ND}=$ Dark Current (electrons/pixel/second) $\mathrm{NR}=$ Read Noise (electrons)

$\mathrm{T}=$ Exposure (seconds)

Note: In the case of EMCCD cameras, the additional EM noise factor of $x \mathrm{I} .4 \mathrm{I}$ must be accounted for.

The approach, therefore, used by camer manufacturers is to a) maximize the light signa reaching the sensor by using a sensor with as high a Quantum Efficiency (QE) as possible and, b) seek to reduce the sources of noise within the system e.g. by cooling.

Why is sensitivity so important? At the most simple level, a more sensitive camera will be more effective at gathering light and producing a high fidelity image at low light levels that are inherent to many fluorescence microscopy applications. However, there are also many practical benefits of greater sensitivity including:

- Reduced laser illumination intensity - keep cells alive throughout study (i.e. suppress phototoxic effects) and also limit dye photobleaching

- $\quad$ Reduced fluorophore concentrations help maintain accurate physiology in living specimens

- Lower exposure times - follow faster processes and get better temporal information

- Get better SNR with TIRF and confocal low light modalities - resulting in better image clarity for techniques that reject out of focus photons
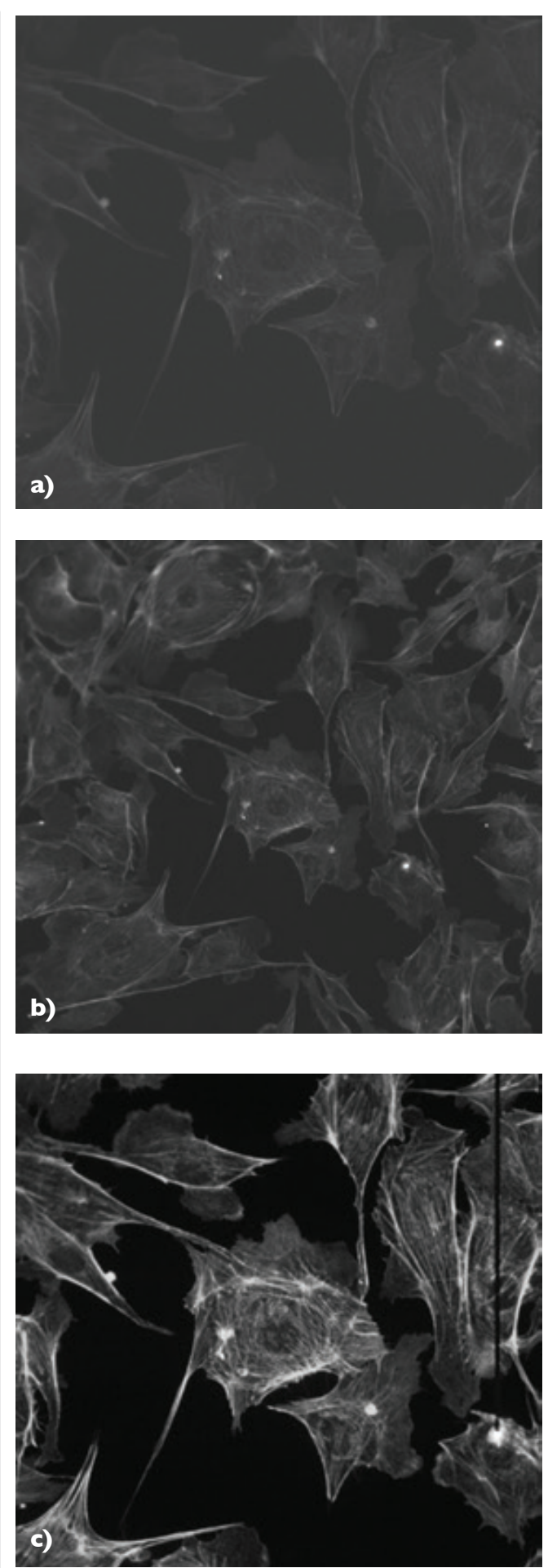

Figure 3: Comparison of a) Zyla, b) Sona, and c) iXon 888 cameras a)-c) have a nucleus diameter of $\sim 20$ microns. The ZYla has been set with $2 \times 2$ binning to bring the smaller pixel up to a comparable effective size as the iXon and Sona. The iXon is set at an EM gain of $\times 300$. Images courtesy of Jeff Oleske, Andor Technolog.
Single Molecule Biophysics - resolve single molecules and spatial positions (Chaurasiya et al. 2018)

There are several ways cameras can be compared to evaluate their sensitivity. This can help us shortlist potential candidates for an application. However, practical comparisons of the camera under the exact conditions of the desired application are likely to be the most definitive.

Firstly, we can compare the cameras using the signal to noise equation; SNR values can typically be found on a cameras' specification sheet. Moreover, the SNRs can be compared and evaluated over different levels of illumination as shown in the graph in Figure I. Therefore, the camera which has the best SNR at the light levels required for the desired application can be determined. For example, in Figure I the SNR are compared for an example of a new backilluminated SCMOS camera (Sona) and a backilluminated EMCCD camera (iXon) at different light levels.

The iXon EMCCD camera still provides a better SNR at light levels below $\sim 10$ photons per pixel, while Sona provides better SNR above this crossover point. It should be noted that for many fluorescence microscopy applications the image data spans the crossover point between SCMOS and EMCCD. In addition, these theoretical measurements are further complicated by the difference in pixel sizes, sensor sizes, and the conversion factor of the camera from photons to electrons.

Alternatively, a calibrated light source and target can be used to compare the images produced at the different light levels. Comparisons of sCMOS against EMCCD using this approach shown in Figure 2 that again demonstrate the superior performance of the EMCCD at low light levels against front (and back) illuminated cameras. Therefore, it is useful to compare the test pattern images of the different cameras at different light levels as this can highlight whether one camera may be better suited to a certain light level.

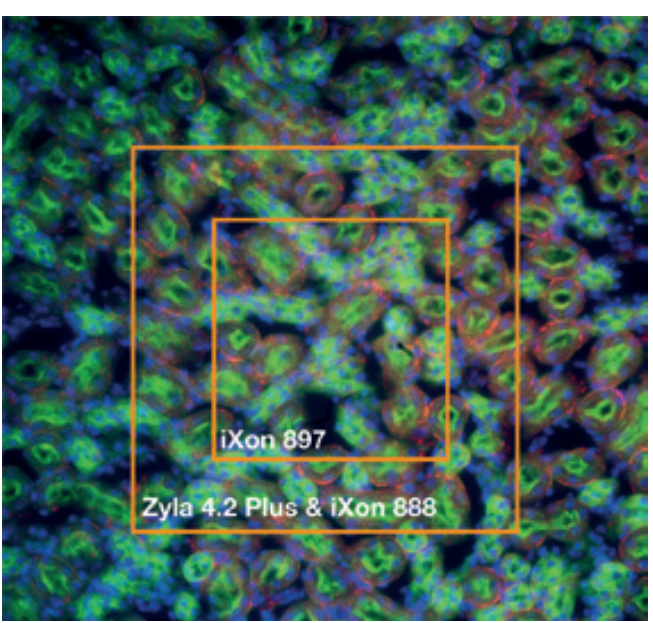

igure 4: Comparison of the field of view of Andor's Sona 4.2B-1/ (FOV $22.5 \times 22.5 \mathrm{~mm}$ ) against other camera models. Orange boxes represent relative size of the respective field of views observable using
he Zyla 4.2 PLUS (I3.3 $13.3 \mathrm{~mm})$ and iXon $888(13.3 \times 13.3 \mathrm{~mm})$ $8897(8.2 \times 8.2 \mathrm{~mm})$ camera models. Kidney cells stained with three flourescent dyes. Glomeruli and convoluted tubules stained with Alexa Fluor 488 (green). Actin stained with red-fluorescent Alexa Fluor 568 phalloidin. Nuclei counterstained with DAPI (blue), Camera Nikon Ti2.

Another method of comparison is simply to set up the cameras for the desired application and compare the results. Indeed, this is often the most practical and effective way to compare the cameras. Consequently, in Figure 3 we compare images of the same sample from the three cameras listed in Table I. In this example, we can clearly see that with the same acquisition settings simulating a light restricted condition, the image taken using the iXon camera gives the best contrast and clarity, largely due to the EM gain. Further, it is evident from Figure 3 that Sona also delivers a superior image to the Zyla. Moreover, from Figures $3 \mathrm{a}$ ), b) and c) the difference in field of view between the cameras is evident, with Sona (b) having a wider field of view of the sample than images taken using either the Zyla or the iXon cameras.

\section{Field of View}

The camera field of view is largely dictated by the sensor size, larger sensors allowing for larger fields of view. However, the field of view must be matched with the microscope to use the available sensor area and avoid vignetting. In order to match the microscope and camera field of view, additional 
magnification in the form of lens couplers is often used, as the field of view will depend on the specific objective lens used.The field of view available through modern microscopes continues to increase, with 22 $\mathrm{mm}$ now being common and models exceeding this now available.

Pixel size is also very important; smaller pixels will help ensure Nyquist sampling and achieve good resolution, whereas, larger pixels are advantageous for sensitivity. Therefore, a compromise on pixel size must be made to optimise the signal to noise and image resolution.

A larger on-sample field of view is of great practica use for a range of studies, as it allows more of the sample to be seen in one field of view and provides more information. Areas where this is of importance include:

- Developmental biology - capture whole embryos, e.g. Zebrafish

- High Content Screening - capture large fields of cells, increase information content

- Tissue Cultures - minimize the need for image stitching, maximize throughput

- Organoids - unravel cell connectivity

- Gene Editing - screen large cell cultures for cells whose genomes have been successfully edited e.g. CRISPR constructs

Figure 4 shows an image of kidney cells taken using Andor's Sona 4.2B-II. Additionally, the respective field of views are overlaid on the image of the Zyla 4.2 PLUS and iXon 888 and 897 camera models. It is clear from Figure 4 that the field of view of the Son camera is the largest of the models compared here. Although Zyla 4.2 PLUS and Sona 4.2B-I I each have a $2048 \times 2048$ sensor, it is the larger II $\mu \mathrm{m}$ pixe size of the Sona that accounts for the difference in their respective field of views. This makes the Sona 4.2B-II the clear choice for whenever the largest field of view is required because more of the sample can be observed in one image. Zyla 4.2 PLUS and iXon 888 have the same sensor size again due to pixel size and array size differences (Table I). The
6.5 um of the Zyla 4.2 PLUS gives the potential for better resolution at magnification objectives e.g. $\times 60$ without the need for lens couplers to match the microscope field of view to the camera field of view.

\section{Speed}

Biological processes can be highly dynamic making the ability to capture events at high speeds the most critical parameter. For example, studies that may require high speed imaging include:

- Ion Signalling - Follow fast calcium wave propagation and calcium sparks with maximum temporal dynamics e.g. in nerve cells (Voleti et al. 2017)

Cell Motility - Speed capability is critica for following cell movement, e.g. sperm cell dynamics.

- Intracellular transport - Fast frame rates can be important to follow intracellular transport dynamics, including membrane dynamics or micro-tubule associated proteins (Monroy et al. 2018)

- Blood flow - perhaps one of the most temporarily challenging applications: speed is of paramount importance (Carboni et $a$. 2016)

\section{- Localization super-resolution e.} SRRF. Many raw images must be rapidly acquired for a single super-resolved output image therefore boosted speed is critical, especially if live cell super-resolution is the true goal (Gustafsson et al. 2016)

As with previous sCMOS models, back-illuminated sCMOS cameras like the Sona allow for imaging at high frame rates, making them well suited to applications such as those listed above. Since the available sensor sizes tend to be relatively large, Region of Interest (ROI) can often be used to provide higher frame rates at the expense of resolution, while 12-bit readout mode can be utilised to accelerate frame rate by up to $2 x$, albeit sacrificing the dynamic range. This is useful for imaging fast processes using low light modalities

Table I: A summary of camera models discussed throughout this article.

\begin{tabular}{|c|c|c|c|c|c|}
\hline $\begin{array}{c}\text { Camera } \\
\text { Model }\end{array}$ & $\begin{array}{c}\text { Camera } \\
\text { Type }\end{array}$ & $\begin{array}{c}\text { Max. } \\
\text { Quantum } \\
\text { Efficiency (\%) }\end{array}$ & Array size & $\begin{array}{c}\text { Pixel Size } \\
\mathbf{( \mu m )}\end{array}$ & $\begin{array}{c}\text { Field of View } \\
\text { (mm) }\end{array}$ \\
\hline $\begin{array}{c}\text { Zyla 4.2 } \\
\text { PLUS }\end{array}$ & $\begin{array}{c}\text { Front- } \\
\text { illuminated } \\
\text { sCMOS }\end{array}$ & 82 & $2048 \times 2048$ & 6.5 & 18.8 \\
\hline $\begin{array}{c}\text { Sona } \\
\mathbf{4 . 2 B}-\text { II }\end{array}$ & $\begin{array}{c}\text { Back-illuminated } \\
\text { sCMOS }\end{array}$ & 95 & $2048 \times 2048$ & 11 & 32 \\
\hline iXon 897 & $\begin{array}{c}\text { Back- } \\
\text { illuminated } \\
\text { EMCCD }\end{array}$ & 95 & $512 \times 512$ & 16 & 11.6 \\
\hline iXon 888 & $\begin{array}{c}\text { Back-illuminated } \\
\text { EMCCD }\end{array}$ & 95 & $1024 \times 1024$ & 13 & 18.8 \\
\hline
\end{tabular}

such as spinning disk confocal or TIRF.

It must be noted that the Zyla 4.2 PLUS with Camera Link connection will deliver higher frame rates than Sona, making the Zyla 4.2 PLUS the best option for where speed is the most important parameter. Some sCMOS camera models such as the Zyla 5.5 and Neo 5.5 have a different mode of operation called Global Shutter. Global Shutter cuts the frame rate in half but the sensor operation mode means that the whole image exposure starts and ends at the same time, eliminating spatial distortion effects. Thus, for experiments in which objects are moving rapidly e.g. for sperm motility, the Zyla 5.5 and Neo 5.5 may be the preferred choice over Rolling Shutter based cameras that use a rolling line-by-line exposure which may produce motion artefacts with fast moving objects.

EMCCD cameras still manage a high frame rate performance, especially when using crop mode, and should be considered for very low light conditions. At low light levels or short exposures times, sCMOS cameras may simply not be sensitive enough to detect the signal. In these cases, EMCCD is still the preferred option despite the on-paper disparity in frame rates.

\section{Conclusion}

The emergence of back-illuminated sCMOS cameras is a welcome development for practitioners in many areas of research, including flourescence microscopy techniques. The back-illuminated sCMOS cameras add increased sensitivity to the arge field of view and fast frame rate performance that front-illuminated sCMOS are well known or. Therefore, the new back-illuminated sCMOS cameras, such as the Sona, are well suited to a diverse range of applications, including high content screening and developmental biology. Moreover they are suitable for studies that require a wide field of view and high sensitivity, so that illumination evels and fluorophore concentrations can be reduced, and exposures shortened for the most accurate measurements of cell physiology.

There are some conditions in which cameras based on the other technologies still offer dvantages over the new back-illuminated sCMOS cameras. The Zyla 4.2 PLUS offers a good overall performance for general fluorescence applications t a lower price point. The Zyla 4.2 PLUS model that uses a Camera Link connection, as opposed to USB 3.0 allows for a higher data transmission rate and so provides a higher frame rate. EMCCD based cameras such as iXon remain the detectors of choice in Andor's range for the most demanding applications due to greater sensitivity even at the lowest light levels. For example, one of the main uses of EMCCD cameras in microscopy has been for single molecule biophysics experiments, and this 
is unlikely to change significantly. EM amplification enables a level of sensitivity that back-illuminate sCMOS cameras still cannot reach.

This article provides an overview of the differen camera technologies available and their relative strengths with regard to sensitivity, field of view and speed. The weighting of these parameters does depend on the specific needs of your research; therefore, it is good practice to evaluate a shortlis of models suitable for your microscopy system so that you can make an informed decision.

\section{References:}

Voleti, V., Li W, Shaik M. A., Wu M., Campos C. P. Wyart C., and Hillman E. M. C.,"Imaging the nervous system at different spatiotemporal scales with SCAPE microscopy," in Optics in the Life Sciences Congress, OSA Technical Digest (online) (Optica Society of America, 2017), paper BrS2B.3.

Chaurasiya K.R., Dame R.T. (2018) Single Molecule FRET Analysis of DNA Binding Proteins. In Peterman E. (eds) Single Molecule Analysis. Methods in Molecular Biology, vol 1665. Humana Press, New York, NY

Monroy B.Y., Sawyer D. L., Ackermann B. E., Borden M.M.,Tan T. C. and Ori-McKenney, K. M. Competition between microtubule-associated proteins directs motor transport. Nature Communications, volume 9,Article number: 1487 (2018)

Erik J. Carboni, Brice H. Bognet, Grant M. Bouchillon, Andrea L. Kadilak, Leslie M. Shor, Michael D. Ward Anson W.K. Ma. Direct Tracking of Particles an Quantification of Margination in Blood Flow, Biophysical Journal, Volume III, Issue 7, 2016, Pages |487-| 495.

Gustafsson, N.; Culley, S.; Ashdown, G.; Owen, D. M.; Pereira, P. M.; Henriques, R. Fast live-cell conventional fluorophore nanoscopy with Image through super-resolution radial fluctuations. Nature Communications 2016, 7, 1247|

\section{About the Authors}

Dr. Alan Mullan: Product Specialist for Microscopy Cameras at Andor Technology.

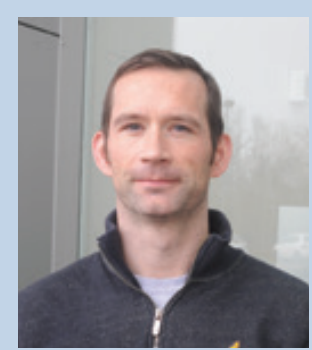

Dr Allan Mullan is the Product Specialist for Microscopy Cameras and has worked at Andor technology for 6 years. This role brings him in touch with the latest developments in camera technology as well as a broad range of microscopy applications. Prior to this he has worked in both the Medical Diagnostics and Pharmaceutical industries. His background in microscopy is built from his work and studies at Queen's University Belfast. He was a post-doctorate Research Fellow focused on the study of phosphate transport and polyphosphate metabolism in microorganisms. Microscopy techniques such as widefield fluorescence, confocal, luminescence and Electron Microscopy were key parts of this work. He received his PhD in Microbiology, also while at Queen's University Belfast.

Dr. Aleksandra Marsh: Technical Author and Copywriter at Andor Technology.

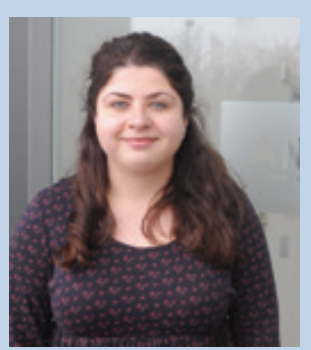

$\mathrm{Dr}$ Marsh recently joined Andor Technology as a Technical Author and Copywriter. Dr Marsh maintains specifications and documentation for the Andor product range, keeping her up to date on camera technologies and microscopy applications. She completed her PhD in Chemistry at the University of Bristol in 2018. During her PhD research in the field of aerosol science she used single particle techniques coupled with Raman spectroscopy, elastic light scattering and brightfield microscopy, to improve understanding of the impact of aerosols on human health and climate.
Focus on a Complete Range of Imaging \& Analysis Solutions

COXEM EM-30 Desktop SEM Range

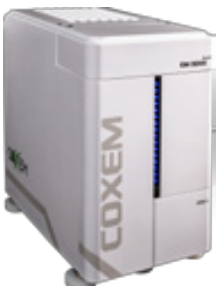

A desktop SEM with advanced, high end features. Options for Tungsten or CeB6 electron sources.

\begin{tabular}{c|l}
\begin{tabular}{c|c} 
High Resolution \\
Imaging
\end{tabular} & $\begin{array}{l}\text { BSE \& SE } \\
\text { Detectors }\end{array}$ \\
\hline
\end{tabular}

COXEM CX2O0 Plus SEM

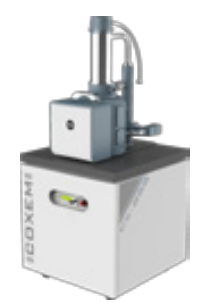

An affordable, powerful SEM in a compact size. Expandable with EDS, WDS, EBSD and more

\begin{tabular}{c|c}
$\begin{array}{c}\text { High Resolution } \\
\text { Imaging }\end{array}$ & $\begin{array}{r}\text { BSE \& SE } \\
\text { Detectors }\end{array}$
\end{tabular}

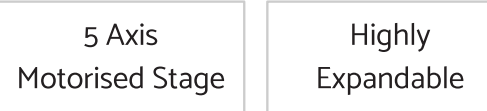

Park Systems NX10 AFM

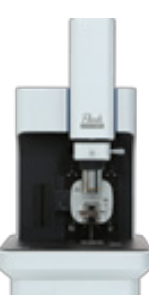

A fast, accurate, and simple AFM. Produce quick, reliable results, with SmartScan 3 step imaging

\begin{tabular}{c|c} 
No XY \\
Scanner Bow
\end{tabular}$\quad \begin{gathered}\text { Low Noise } Z \\
\text { Detector }\end{gathered}$

True Non-Contact

Extensible \& Expandable

Visit CN Tech at mmc2O19 to experience demonstrations of these latest innovations in SEM and AFM technology, plus much more.

Specialists in surface analysis and materials testing www.cntech.co.uk

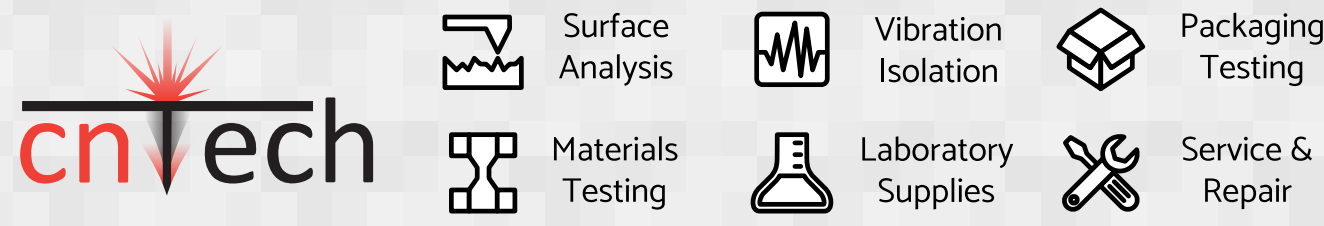

